\title{
A Revised Inverse Data Envelopment Analysis Model Based on Radial Models
}

\author{
Xiaoyin $\mathrm{Hu}^{1,2, *,+} \mathbb{C}^{\mathbb{C}}$, Jianshu $\mathrm{Li}^{1,2,+},{\text { Xiaoya } \mathrm{Li}^{1} \text { and Jinchuan Cui }}^{1}$ \\ 1 Academy of Mathematics and Systems Science, Chinese Academy of Sciences, Beijing 100190, China; \\ ljs@amss.ac.cn (J.L.); xyli@amss.ac.cn (X.L.); cjc@amss.ac.cn (J.C.) \\ 2 School of Mathematical Sciences, University of Chinese Academy of Sciences, Beijing 100049, China \\ * Correspondence: hxy@amss.ac.cn \\ + These authors contributed equally to this work.
}

Received: 17 April 2020; Accepted: 12 May 2020; Published: 15 May 2020

\begin{abstract}
In recent years, there has been an increasing interest in applying inverse data envelopment analysis (DEA) to a wide range of disciplines, and most applications have adopted radial-based inverse DEA models. However, results given by existing radial based inverse DEA models can be unreliable as they neglect slacks while evaluating decision-making units' (DMUs) overall efficiency level, whereas classic radial DEA models measure the efficiency level through not only radial efficiency index but also slacks. This paper points out these disadvantages with a counterexample, where current inverse DEA models give results that outputs shall increase when inputs decrease. We show that these unreasonable results are the consequence of existing inverse DEA models' failure in preserving DMU's efficiency level. To rectify this problem, we propose a revised model for the situation where the investigated DMU has no slacks. Compared to existing radial inverse DEA models, our revised model can preserve radial efficiency index as well as eliminating all slacks, thus fulfilling the requirement of efficiency level invariant. Numerical examples are provided to illustrate the validity and limitations of the revised model.
\end{abstract}

Keywords: data envelopment analysis; efficiency index; multi-objective programing; slacks

MSC: 65K99; 90C11; 05A05

\section{Introduction}

Data envelopment analysis (DEA) is a linear programming (LP) based method to assess the efficiency level of decision-making units (DMUs) with multiple inputs and outputs. DEA is useful for empirically measuring the productive efficiency of DMUs [1], for example, health care [2,3], education [4], economies [5-7], banks [8,9], etc. The first DEA model, the Charnes, Cooper, and Rhodes (CCR) model [10], evaluates the efficiency level of each DMU in two aspects, radial inefficiency and mix inefficiency. The radial inefficiency is measured by a scalar named radial efficiency index, while the mix inefficiency is identified by slacks of the corresponding LP. This evaluation process indicates that not only radial efficiency index but also slacks should be considered to measure the efficiency level. The CCR model and other DEA models with similar evaluation process are referred to as radial DEA models in the literature.

Inverse DEA is an extension of DEA that has been extensively studied and applied during the last two decades. It has been proved useful in various real-life applications as it enhanced conventional DEA with the function of making predictions. The underlying assumption of inverse DEA is that, during the period of interest, the efficiency level of DMUs remains the same [11]. Under this assumption, for example, if inputs of a DMU alter to a different level, then it will adjust its outputs so that the DMU's efficiency status remains unchanged. Inverse DEA aims to find such outputs level 
(inputs level if a target outputs level is preassigned). An inverse DEA model has two key features: its base DEA model and the efficiency measure it has adopted. In this paper, we only study inverse DEA models that are based on radial DEA models and refer to them as radial inverse DEA models; in fact, most existing inverse DEA models belong to this category. As for the measure of efficiency level, all radial inverse DEA models only consider radial efficiency index and neglect slacks. However, as the efficiency evaluation process of radial DEA models suggests, the efficiency level of DMUs is measured by not only radial efficiency but also slacks. Therefore, current radial inverse DEA models may fail to meet the requirement of efficiency invariant, and the predictions given by these models are not always reliable. Though this disadvantage can severely undermine the credibility of inverse DEA models, to our best knowledge, it has not been noticed in literature. In this paper, we attempt to fill this gap by assessing the influence of this disadvantage on inverse DEA and giving revision for some situations.

Inverse DEA was initialized by Zhang and Cui [12]. Zhang and Cui proposed a model to estimate the inputs level of a DMU given outputs' increments, under the constraint that the Constant Return to Scale (CRS) index of the DMU is unchanged. Wei et al. [11] generalized inverse DEA to preserve various return to scale (VRS), non-increasing return to scale(NIRS) and non-decreasing return to scale (NDRS) index. They also introduced a multi-objective linear programming (MOLP) technique to formalize inverse DEA models as well as the scalarization method to solve the corresponding MOLP. Based on the paradigm of [11], various extensions of inverse DEA have been developed. In general, these extensions can be classified into three categories. Extensions in the first category aim to complement uncovered scenarios of inverse DEA based on the classic radial DEA models. See, e.g., [13-16]. Recently, Zhang and Cui [17] integrated inverse DEA models of this category into 12 different cases. The second category consists of works that extend inverse DEA by introducing additional factors such as preference cone [18], undesirable outputs or inputs [19,20], and cost efficiency [21]. The last category includes studies that relax some requirements of conventional inverse DEA. For example, in [22], the constraint of unchanged efficiency level is generalized to a target efficiency level, and Lim [23] considered frontier shift. Extensions in this category still follow the principle of inverse DEA and also resort to the MOLP technique. Besides theoretical developments, practically there has been an increasing interest in using inverse DEA to solve problems across a wide range of disciplines. Applications of inverse DEA include but not limited to: revenue setting [24], agricultural resources demand estimation [25], enterprise reconstruction [26-28], carbon abatement allocation [29,30], and sustainability analysis [31]. However, despite the variations, all studies mentioned above are based on radial DEA models and employ radial efficiency index as the only efficiency measure, which means that all of them have used an incomplete efficiency measure. For example, Emrouznejad et al. [30] allocated carbon emission abatement across 31 sub-level industrial manufacturing in China based on a radial model. With an abatement on about $9 \%$ of the total carbon emission, their proposed radial model suggests cutting $95 \%$ of the total carbon emission abatement in one sub-level industries (Manufacture of Non-metallic Mineral Products), which is unreasonable in practice. There are a few works based on the non-radial DEA model [32,33], where an inverse problem is designed for the enhanced Russel model. However, solving these problems is difficult due to the non-convexity in the objective functions and constraints in these problems. Radial models are widely applied in allocation problems since many of them can be reshaped as linear programming. Due to the incomplete efficiency measure problem in a radial model, it is important to determine the cases where these radial models can be efficiently and reasonably applied. In addition, it is of great importance to develop a revised inverse radial DEA model to fix the incomplete efficiency measure problem.

The contributions of this paper are two-fold. Firstly, we show that neglecting slacks in radial inverse DEA can lead to unreasonable results. We give a numerical example to support this assertion, where an inverse DEA model predicts outputs to decrease while inputs increase. When the investigated DMU has non-zero slacks, this kind of failure is inevitable. Secondly, for DMUs without mix inefficiency, we propose a revised inverse DEA model that can satisfy the requirement of efficiency invariant. 
The revised model is constructed by supplementing an extra constraint to confine DMUs to possess no mix inefficiency after the change. We develop an algorithm to solve the revised model in a simplex-liked manner. In our numerical examples, Algorithm 1 computes all pareto solutions for our test examples, where their radial efficiency indexes are unchanged and the mix inefficiency is avoided. Numerical experiments illustrate that our algorithm can keep the radial efficiency level of DMUs unchanged during the prediction. In addition, the traditional inverse DEA [11] requires prefixed relative weights of each output, which may cause manipulating the results according to one's own preference. Our model avoids prior assumptions about relative weights of each input and output while accessing DMUs' relative efficiency. The weights prescribed by the DEA model are derived directly from the data and each DMU is evaluated in its best possible light. However, the premise of our model leads to the limitations, and our model can only predict DMUs with no mix inefficiency during the two-phase procedure. In addition, for DMUs that meet this condition, the predictable range is also limited. As a conclusion, these numerical examples further show the validity and limitations of our method.

This paper is organized as follows. Section 2 provides some preliminary materials including radial-based DEA models, two-phase method, properties of MOLP, and inverse DEA models. We also state our assumptions in this section. A numerical counterexample is given in Section 3. Section 4 presents the revised inverse DEA model and the simplex algorithm to solve it. Two numerical illustrations are also exhibited in this section. Finally, Section 5 concludes the paper and suggests future studies.

\section{Preliminaries}

\subsection{Radial DEA Models}

We consider a system of $n$ DMUs, where inputs and outputs matrices are $X=\left(x_{i j}\right) \in \mathbb{R}^{m \times n}, X \geq 0$ and $Y=\left(y_{i j}\right) \in \mathbb{R}^{s \times n}, Y \geq 0$, respectively. Let $\lambda=\left(\lambda_{1}, \ldots, \lambda_{n}\right) \in \mathbb{R}^{n}$, the general production possible set (PPS) be defined as:

$$
T=\left\{(x, y): \sum_{j=1}^{n} x_{j} \lambda_{j} \leq x, \sum_{j=1}^{n} y_{j} \lambda_{j} \geq y, \lambda \in \Lambda\right\}
$$

where $\Lambda$ is one of the following:

$1 \Lambda_{C}=\{\lambda: \lambda \geq 0\}$, corresponds to constant return to scale (CRS),

$2 \Lambda_{V}=\left\{\lambda: \sum_{j=1}^{n} \lambda_{j}=1, \lambda \geq 0\right\}$, corresponds to various return to scale (VRS),

$3 \Lambda_{N I}=\left\{\lambda: \sum_{j=1}^{n} \lambda_{j} \leq 1, \lambda \geq 0\right\}$, corresponds to non-increase return to scale (NIRS),

$4 \Lambda_{N D}=\left\{\lambda: \sum_{j=1}^{n} \lambda_{j} \geq 1, \lambda \geq 0\right\}$, corresponds to non-decrease return to scale (NDRS).

Radial DEA models can be input-oriented or output-oriented, for simplicity, we confined our study to input-oriented models in this paper. Nevertheless, our results are valid for output-oriented models with proper modifications.

Input-oriented models evaluate DMUs' efficiency level through a two-phase procedure [34]. Denote the investigated DMU as $\mathrm{DMU}_{0}$, and its inputs and outputs as $X_{0}$ and $Y_{0}$, the two-phase procedure proceeds as follows:

\section{Phase 1}

Solve:

$$
\begin{aligned}
\left(\mathrm{DLP}_{\mathrm{I}}\right) & \min \theta \\
\text { s.t. } & \left(\theta \mathrm{X}_{0}, Y_{0}\right) \in T \\
& \theta \in \mathbb{R}
\end{aligned}
$$

let the optimal solution be $\theta^{*}$. 


\section{Phase 2}

Insert the value of $\theta^{*}$ to solve the following LP :

$$
\begin{aligned}
\left(\operatorname{MAX}_{\mathrm{S}}\right) & \max _{\lambda, s^{-}, s^{+}} \omega=w_{x}^{T} s^{-}+w_{y}^{T} s^{+} \\
\text {s.t. } & s^{-}=\theta^{*} x_{o}-X \lambda \\
& s^{+}=Y \lambda-y_{o} \\
& \lambda \in \Lambda, s^{-} \geq 0, s^{+} \geq 0
\end{aligned}
$$

where $w_{x} \in \mathbb{R}^{m}$ and $w_{y} \in \mathbb{R}^{s}$ are arbitrary positive weight vectors. With a little abuse of nation, in the rest of this paper, we use $\left(\theta^{*}, s^{-}, s^{+}\right)$to denote the results of phase 1 and phase 2 .

Phase 1 attempts to reduce the inputs of $\mathrm{DMU}_{\mathrm{o}}$ proportionally (radially) as much as possible while keeping the changed $\mathrm{DMU}_{\mathrm{o}}$ remaining in $T$. The optimal value solution $\theta^{*}$ is called radial efficiency index of $\mathrm{DMU}_{\mathrm{o}}$. Phase 2 seeks to find maximal possible inputs excesses and outputs shortfalls while maintaining $\theta^{*}$. Slack variables, $s^{-}$and $s^{+}$, reflect these excesses and shortfalls, respectively. If non-zero slacks appear in the optimal solution, it indicates that there exists an extra source of inefficiency besides radial efficiency, and this kind of inefficiency is referred to as mix inefficiency. We will use non-zero slacks and mix inefficiency interchangeably in the rest of this paper.

Based on the results of these two phases, we define the strongly and weakly efficient as:

Definition 1. If an optimal solution of above two-phase procedure satisfies $\theta^{*}=1$ and all slacks are zero, then $\mathrm{DMU}_{\mathrm{O}}$ is called strongly efficient;

If $\theta^{*}=1$ and there are non-zero slacks, then $\mathrm{DMU}_{\mathrm{o}}$ is called weakly efficient.

Remark 1. If two DMUs have the same radial efficiency index $\theta^{*}$ but different slacks, their efficiency is different.

\subsection{Multiple Objective Linear Programming}

Almost all inverse DEA models resort to MOLP. Therefore, it is necessary to review some properties of MOLP before we proceed to inverse DEA models. A general MOLP can be represented as:

$$
\begin{array}{rl}
V-\max _{x} & y=C x \\
\text { s.t. } & A x=b \\
& x \geq 0
\end{array}
$$

where $V$-max indicates it is a vector to be maximized, and $C \in \mathbb{R}^{p \times n}$ means there are $p$ objectives, hence we have $y \in \mathbb{R}^{p}$.

By convention, we always assume that:

Assumption 1. The p objects of above MOLP can not be optimized at the same time.

Assumption 2. For any obtainable objective vector $y$, the corresponding feasible solution $x$ is unique.

The solutions' concepts of MOLP are defined as follows.

Definition 2 (Strongly efficient Solution). A feasible solution $x$ is a strong efficient solution of the MOLP above if there is no feasible solution $x^{\prime}$ such that $y_{i}^{\prime} \geq y_{i}$ for all $i=1,2, \ldots, p$ and $y_{i}^{\prime}>y_{i}$ for at least one $i$.

Definition 3 (Weakly efficient Solution). A feasible solution $x$ is a strong efficient solution of the MOLP above if there is no feasible solution $x^{\prime}$ such that $y_{i}^{\prime}>y_{i}$ for all $i=1,2, \ldots, p$.

Since all properties we are going to present are valid for both solution concepts, in the rest of this subsection, we will not differentiate them and refer to them collectively as efficient solutions. Denote the feasible space of MOLP as $\mathcal{X}, \mathcal{X}_{E}$ as the subset of $\mathcal{X}$ that contains all efficient solutions. 
Definition 4. Face $\mathcal{F} \in \mathcal{X}$ is called efficient face if $\mathcal{F} \in \mathcal{X}_{E}$. If there is no efficient face $\mathcal{F}^{\prime}$ that $\mathcal{F} \in \mathcal{F}^{\prime}$, it is called maximal efficient face.

For the structure of strongly (weakly) efficient solutions of MOLP, we have:

Proposition 1. $\mathcal{X}_{E}$ is the union of maximal efficient faces.

Proposition 2. $\mathcal{X}_{E}$ is connected.

MOLP and LP have a feasible region of the same form as polyhedrons. In the simplex algorithm of LP, feasible solutions of LP that correspond to extreme points of the polyhedron are called basic feasible solutions (BFSs), and basis related to BFSs is called the feasible basis (FB). Analogously, we can define:

Definition 5 (Basic Efficient Solution, Efficient Basis). An efficient solution of an MOLP is called Basic Efficient Solution (BES) if it corresponds to an extreme point of $\mathcal{X}_{E}$. Basis that corresponds to BESs is called Efficient Basis (EB).

In addition, adjacent relations as:

Definition 6 (Efficient Basis Adjacent, Neighbor). Efficient basis $E B_{1}$ and $E B_{2}$ are called adjacent if they are different in a single component. $E B_{1}$ and $E B_{2}$ are neighbors of each other.

MOLP can be solved by the multiple objective simplex algorithms; see, e.g., [35,36]. These algorithms can locate all BES of MOLP and give a complete description of $\mathcal{X}_{E}$. The key procedure of these algorithms is the efficient pivot rule that can probe among all BESs, and each pivot is usually achieved by solving a linear programming.

\subsection{Inverse DEA Model}

Current radial inverse DEA models seek to estimate inputs/outputs while maintaining the radial efficiency index. In this paper, we only study the case where DMU of interest is inefficient, which is $\theta_{o}<1$. Consider the inverse DEA model given in [17] for an inefficient DMU denoted as $\mathrm{DMU}_{\mathrm{o}}$ :

$$
\begin{aligned}
\text { (Intputx) } & V \text {-max } \beta \\
\text { s.t. } & \left(\theta_{o} \alpha, \beta\right) \in T \\
& \beta \in \mathbb{R}^{s}
\end{aligned}
$$

where the name Input $X$ indicates that this model is input-oriented and assigned change is on the input side; $\alpha$ is the inputs after change; $\beta$ is the outputs to be found; and $\theta_{0}<1$ is the radial index of $\mathrm{DMU}_{\mathrm{o}}$.

Model Input $x$ aims to find outputs that can preserve radial index $\theta_{0}$ when inputs change to $\alpha$. Wei et al. [11] introduced the MOLP technique to solve models like Inputx. In their work, a weakly efficient solution of MOLP is used as a prediction result. Later, Hadi-Vencheh et al. [15] found a counterexample of the method in [11] and suggested to use a strong solution instead of a weak solution of MOLP. Furthermore, Zhang and Cui [17] gave a detailed description of the necessary conditions to use inverse DEA models. Without loss of generality, we adopt the method of Hadi-Vencheh et al. [15] in this paper and assume the conditions mentioned in [17] are satisfied.

Finally, we have:

Theorem 1 ([11,15,17]). Suppose $(\beta, \lambda)$ is a feasible solution of model Inputx. Then, new DMU with production combination $(\alpha, \beta)$ has the same radial efficiency index as $\mathrm{DMU}_{\mathrm{o}}$ if $(\beta, \lambda)$ is a strongly efficient solution of Inputx. 
The strongly efficient solution can be found using a scalarization technique introduced in [11].

\section{Drawbacks of Inverse DEA on Radial Models}

\subsection{A Numerical Counterexample}

We use model Input $x_{X}$ under the variable return to scale (VRS) assumption to present the numerical example. Table 1 exhibits the dataset, and the results of the corresponding two-phase procedure are listed in Table 2.

Table 1. Production dataset.

\begin{tabular}{lccccc}
\hline & DMU $_{\mathbf{1}}$ & $\mathbf{D M U}_{\mathbf{2}}$ & $\mathbf{D M U}_{\mathbf{3}}$ & $\mathbf{D M U}_{\mathbf{4}}$ & $\mathbf{D M U}_{\mathbf{5}}$ \\
\hline Input 1 & 4 & 6 & 1 & 4 & 2 \\
Input 2 & 3 & 6 & 1 & 2 & 4 \\
Output 1 & 1 & 4 & 3 & 4 & 4 \\
Output 2 & 1 & 3.5 & 4 & 5 & 2 \\
\hline
\end{tabular}

Table 2. Results of two phase procedure.

\begin{tabular}{lcccccc}
\hline & $\mathbf{D M U}_{\mathbf{1}}$ & $\mathbf{D M U}_{\mathbf{2}}$ & $\mathbf{D M U}_{\mathbf{3}}$ & $\mathbf{D M U}_{\mathbf{4}}$ & $\mathbf{D M U}_{\mathbf{5}}$ & $\mathbf{D M U}_{\mathbf{1}}^{\prime}$ \\
\hline Efficiency & $1 / 3$ & $1 / 2$ & 1 & 1 & 1 & $1 / 3$ \\
$s_{1}^{-}$ & $1 / 3$ & 0 & 0 & 0 & 0 & 0 \\
$s_{2}^{-}$ & 0 & 0 & 0 & 0 & 0 & 0 \\
$s_{1}^{+}$ & 2 & 0 & 0 & 0 & 0 & 0 \\
$s_{2}^{+}$ & 3 & 0 & 0 & 0 & 0 & 0 \\
\hline
\end{tabular}

Suppose the inputs of $\mathrm{DMU}_{1}$ decrease to $\alpha=(3,3)$, and the goal is to find outputs $\beta$ that can maintain the radial efficiency index.

It can be checked that $\beta=(3,4)$ is a solution that satisfies model Input ${ }_{X}$, as DMU with inputs level $\alpha$ and outputs level $\beta$ has radial efficiency index $1 / 3=\theta_{1}$. However, this result does not conform to common experience. While the inputs of $\mathrm{DMU}_{1}$ are assigned to decrease, the inverse DEA model predicts the outputs shall increase as $(3,4)>(1,1)$. This example reveals that radial inverse DEA models can give unreasonable results.

Furthermore, let the DMU with production combination $(\alpha, \beta)$ be $\mathrm{DMU}_{1}^{\prime}$ and perform the two-phase procedure on it. The result can be found in the last column of Table 2, where all slacks are zero. Note that $\mathrm{DMU}_{1}$ has no mix inefficiency after the change, whereas, before the change, $\mathrm{DMU}_{1}$ possesses both radial and mix inefficiencies. Since the radial inefficiency is preserved during the process, the overall efficiency level of $\mathrm{DMU}_{1}$ improved, which violates the inverse DEA's requirement of efficiency invariant.

\subsection{Theoretic Analysis on the Reason for Drawbacks}

The unreasonable result is not an occasional failure. Instead, it is the inevitable consequence of neglecting mix inefficiency. In this subsection, we extend the counterexample to a general case to support this assertion.

Theorem 2. Suppose the optimal solution of two-phase procedure of $\mathrm{DMU}_{\mathrm{o}}$ is $\left(\theta^{*}, s^{-}, s^{+}\right)$. If $x_{0}$ is reduced to $\alpha$ and $x_{0}-\frac{1}{\theta^{*}} s^{-} \leq \alpha \leq x_{0}$ and $\beta=y_{0}+s^{+}$, then $(\alpha, \beta)$ has radial efficiency index $\theta^{*}$.

Proof. Denote DMU with production profile $(\alpha, \beta)$ as $\mathrm{DMU}_{\mathrm{o}}^{\prime}$.

By our assumption that $\theta^{*}<1$ and according to Lemma 1 in [17], we have $\lambda_{o}^{*}=0$. For inputs, $j=1, \ldots, m$ : 


$$
\begin{aligned}
\sum_{i=1}^{n} x_{j i} \lambda_{i} & =\theta^{*} x_{j o}-s_{j}^{-} \\
& =\theta^{*}\left(x_{j o}-\frac{1}{\theta^{*}} s_{j}^{-}\right) \\
& \leq \theta^{*} \alpha
\end{aligned}
$$

For outputs, $j=1, \ldots, s$ :

$$
\begin{aligned}
\sum_{i=1}^{n} y_{j i} \lambda_{i} & =y_{j o}-s_{j}^{+} \\
& =\beta
\end{aligned}
$$

By Equations (6) and (7), $\left(\theta^{*}, \lambda^{*}\right)$ is feasible to model DLP $P_{\mathrm{I}}$. Therefore, $\theta^{*} \geq \theta^{\prime}$, where $\theta^{\prime}$ is the efficiency index of $\mathrm{DMU}_{\mathrm{o}}^{\prime}$.

Now assume by contradiction that $\theta^{*}>\theta^{\prime}$.

Let $\left(\theta^{\prime}, \lambda^{\prime}, s^{-^{\prime}}, s^{+^{\prime}}\right)$ be the optimal solution of two-phase procedure of $\mathrm{DMU}_{\mathrm{o}}^{\prime}$. We have :

$$
\begin{aligned}
& X \lambda^{\prime} \leq \theta^{\prime} \alpha<\theta^{*} \alpha \leq \theta^{*} x_{o} \\
& Y \lambda^{\prime} \geq \beta \geq y_{o} .
\end{aligned}
$$

The strict inequality in Equation (8) reveals that there exists $\lambda^{\prime}$ that $X \lambda^{\prime}<\theta^{*} x_{0}$; as a consequence, $\theta^{*}$ is suboptimal to original model, which is a contradiction.

Base on Theorem 2, we can obtain Corollary 1 that characterizes the counterexample.

Corollary 1. Suppose the optimal solution of two-phase procedure of $\mathrm{DMU}_{\mathrm{o}}$ is $\left(\theta^{*}, \lambda^{*}, s^{-*}, s^{+*}\right)$. If $x_{0}$ is reduced to $\alpha$ and $\alpha=x_{0}-\frac{1}{\theta} s^{-*}$ and $\beta=y_{0}+s^{+*}$, then $(\alpha, \beta)$ has radial efficiency index $\theta^{*}$.

The key observation of the above counterexamples is that current inverse DEA models allow DMUs' inputs and outputs to vary within the range of slacks, even in a controversial way. Such ambiguousness will undermine inverse DEA's credibility as a prediction and production planning tool. Take resource allocation, for instance. Because the goal is commonly set to maximize overall outputs, the problem reported in this section would lead to an overly optimistic result as if outputs of some DMUs can increase for free. Therefore, results given by current radial inverse DEA models should be reexamined, especially when slacks are comparatively notable.

A similar phenomenon has been reported in the literature but interpreted differently. Jahan-shahloo et al. [13] studied the situation where a certain amount of inputs can be reduced without altering the radial efficiency index. They regarded reduced inputs as extra resources instead of sources of inefficiency. However, this interpretation implies that DMUs choose to waste some inputs resources on purpose when making production decisions, contradicting the conventional assumption that DMUs are rational.

Instead, we attribute the counterexamples to the altered efficiency status of DMUs, as we explained in the preceding subsection. Furthermore, since current radial DEA models neglect slacks while evaluating the efficiency of DMUs, it is unclear if they have preserved the efficiency status of DMUs as required. As a result, even if the undesirable effects do not show up, results given by current radial inverse DEA models are nevertheless questionable.

We finally remark that, although our counterexample is about outputs' estimation under inputs' decrease, it hints at other scenarios where solutions given by inverse DEA models can be controversial. For example, consider an opposite process of our example to find inputs' level under outputs' increase, our analysis is also valid. Similar problems can be found in other scenarios or output-orientated models. 


\section{A Revised Inverse DEA Model}

\subsection{A Revised Inverse DEA Model}

Failures reported in Section 3 reflect the conflict between the inverse DEA's requirement of evaluating efficiency level explicitly and the fact that classic radial DEA models do not provide a unified efficiency measure. This conflict is irreconcilable for general situations. Nevertheless, we can avoid it in a particular situation, where DMU of interest has no mix inefficiency. In this situation, the radial efficiency index is a comprehensive measure of efficiency. Thus, the requirement of inverse DEA can be fulfilled by preserving radial efficiency index and restraining all slacks in the corresponding two-phase procedure to be zero.

We adopt the same notations in Section 3. Denote $\mathrm{DMU}_{\mathrm{o}}$ as the investigate $\mathrm{DMU}$, whose original inputs and outputs are $\left(x_{0}, y_{0}\right)$ with radial efficiency index $\theta_{0}$ and no mix inefficiency.

Since conventional inverse DEA models have already solved the problem of maintaining radial efficiency index, we only need to consider slack variables. The following lemma shows that slack variables can be handled via model Input ${ }_{X}$.

Lemma 1. Suppose the inputs of $\mathrm{DMU}_{\mathrm{o}}$ change to $\alpha$, and $x=\left(\beta, \lambda^{T}, s^{-}, s^{+}\right)$is a strongly efficient solution of model Inputx. Then, model $M A X_{S}$ with production profile $(\alpha, \beta)$ has optimal value zero if and only if $s^{-}=0$, $s^{+}=0$.

Proof. Notice that any solution of Input $_{X}$ is also feasible to MAX . $_{\text {. }}$

For the side of the theorem, suppose $\operatorname{MAX}_{\mathrm{S}}$ has an optimal solution $\tilde{x}=\left(\tilde{\lambda}, \tilde{s}^{-}, \tilde{s}^{+}\right)$that some slack variables are not equal to zero.

Firstly, we prove that, in any optimal solution of $\mathrm{MAX}_{\mathrm{S}}, \tilde{\mathrm{s}}^{+}=0$.

By the constraints of model $\mathrm{MAX}_{S}$, we know $\beta=Y \tilde{\lambda}-\tilde{s}^{+}$; then, $\left(Y \tilde{\lambda}, \tilde{\lambda}, \tilde{s}^{-}, 0\right)$ is feasible to Input $x$. However, if $\tilde{s}^{+}>0$, we will have $Y \tilde{\lambda}>\beta$, which is not possible since $\beta$ is strongly efficient. An implication of this fact is that any optimal solution $\hat{x}$ of MAX is also a strongly efficient solution of Input ${ }_{X}$; this is obvious since, if $\tilde{s}^{+}=0, Y \tilde{\lambda}=\beta$ is strongly efficient.

Secondly, we establish that $\tilde{s}^{-}=0$.

Any convex combination of $\hat{x}$ and $x$ is also strongly efficient, with the same objective vector $\beta$. This leads to a contradiction to Assumption 2; therefore, we must have $\tilde{s}^{-}=0$. Hence, the if side holds.

The only if side of this theorem is obvious.

This lemma aids us to transform Inputx into a more compact representation:

Corollary 2. Model Input $\mathrm{x}$ is equivalent to model below:

$$
\begin{array}{cl}
\text { Input } \left._{\mathrm{X}}^{\prime}\right) & V-\max _{\lambda, s^{-}} Y \lambda \\
\text { s.t. } & X \lambda+s^{-}=\theta \alpha \\
& \lambda \in \Lambda
\end{array}
$$

With the help of Lemma 1 and Corollary 2, our goal is specified as: to find a strongly efficient solution MOLP Input ${ }_{X}^{\prime}$ which has only zero slacks. Since there is no guarantee that such a strongly efficient solution always exists, the revised model should be able to decide whether a solution satisfies the requirements that exists at all.

Now, we are in the position to present our revised inverse DEA model:

$$
\begin{aligned}
\text { (R-Input } & \min _{\lambda, s^{-}} \delta=e^{T} s^{-} \\
\text {s.t. } & \left(\lambda, s^{-}\right) \in \mathcal{X}_{E},
\end{aligned}
$$

where $e \in \mathbb{R}^{m}$ is unit vector. If the optimal value of R-Inputx is greater than 0 , it indicates that 
there is no outputs level $\beta$ that can satisfy radial efficiency invariant and zero slacks at the same time. Otherwise, if R-Input $X_{X}$ has an optimal value that is equal to 0 , then the desired outputs can be computed by $Y \lambda^{*}$, where $\left(\lambda^{*}, 0\right)$ is a optimal solution.

For the correctness of this model, we have:

Theorem 3. Model Input ${ }_{X}^{\prime}$ has strongly efficient solution with only zero slacks if and only if model $R$-Input has optimal value zero.

Proof. R-Inputx is bounded below by 0 as $s^{-} \geq 0$, therefore it has optimal value zero only when $s^{-}=0$. The rest of the proof is obvious.

Model R-Input ${ }_{X}$ is defined over the strongly efficient solution $\mathcal{X}_{E}$, which can be problematic as we do not have a complete representation of $\mathcal{X}_{E}$. Furthermore, representing $\mathcal{X}_{E}$ explicitly can be computationally prohibitive, as such representation could include faces with number exponential to $n, m, s$ [37]. We developed a simplex algorithm for R-Inputx to avoid enumerating all strongly efficient solutions. See Algorithm 1.

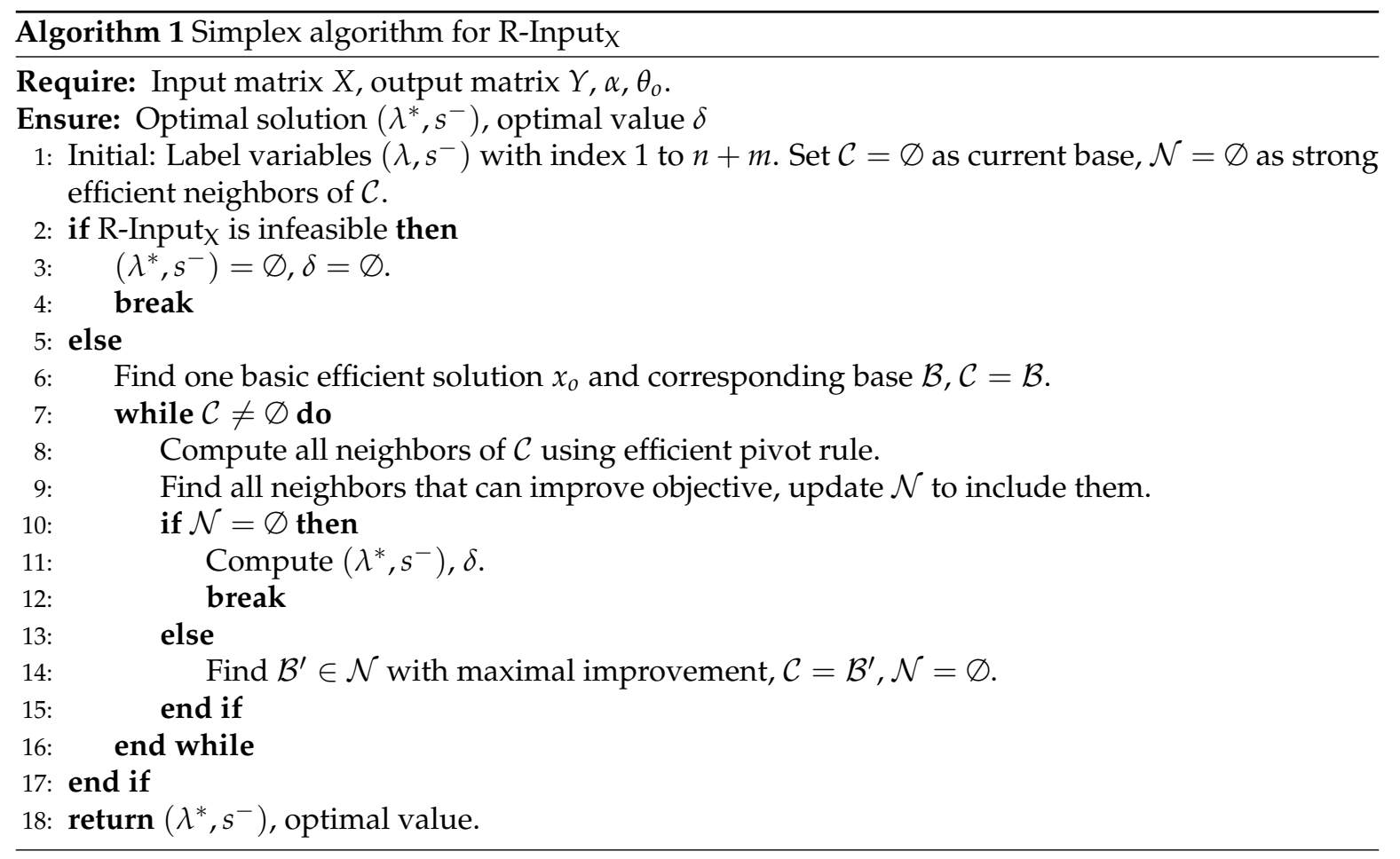

The basic idea of this algorithm is to improve the objective of R-Input $\mathrm{X}$ by pivoting among efficient basis. If the model is feasible, we can find an initial basic efficient using methods like [38]. The efficient pivot rules can recognize or exclude a neighbor of the current efficient base as an adjacent BES by solving a linear programming (We are not going to give a complete description of these rules, knowing they are effective is sufficient for the purpose here. Details of these pivoting rules can be found in various sources like [36,37] ). Therefore, Algorithm 1 proceeds in a simplex algorithm manner; at each step, it seeks to improve the objective value by pivoting to an adjacent base. The maximal improvement protocol, which is the Bland's rule [39], guarantees that the procedure is circling free in case of degeneracy.

Now, we are going to justify the algorithm. In Algorithm 1, we only probe BESs of Input ${ }_{X}^{\prime}$, which is only a subset of all strongly efficient solution $\mathcal{X}_{E}$. Lemma 2 confirms that considering only BESs is sufficient. 
Lemma 2. If there exists a strongly efficient solution $x$ of Input ${ }_{X}^{\prime}$ that $s^{-}=0$, then there exist BES that $s^{-}=0$.

Proof. Notice that the efficient face of MOLP is a polyhedron and any strongly efficient solution must be on some efficient faces. Suppose $x$ is on face $\mathcal{F}$.

If $\mathcal{F}$ is bounded, then $x$ can be represented as a convex combination of the extreme points of $\mathcal{F}$. Denote the extreme points as $e_{1}, \ldots, e_{k} ;$ by representation theorem of a polyhedron, there exist nonnegative real numbers $\mu_{i}, i=1, \ldots, k$ such that $0 \leq \mu_{i} \leq 1, i=1, \ldots, k, \sum_{i=1}^{k} \mu=1$ satisfies:

$$
\left(\lambda^{T}, 0,0\right)^{T}=\sum_{i=1}^{k} \mu_{i} e_{i} .
$$

Since $s^{-}=0, s^{+}=0$, the corresponding parts of extreme points $e_{i}, i=1,2, \ldots, l$ must be zero.

If $\mathcal{F}$ is unbounded, then $x$ can be represented as a combination of extreme points and extreme rays. Let the extreme points be $e_{1}, \ldots, e_{k}$ and extreme rays be $d_{1}, \ldots, d_{l}$. Then, there are nonnegative real numbers $\mu_{i}, i=1, \ldots, k$ and positive real numbers $v_{i}, i=1, \ldots, l$ such that $0 \leq \mu_{i} \leq 1, i=1$, $\ldots, k, \sum_{i=1}^{k} \mu=1$ satisfy:

$$
\left(\lambda^{T}, 0,0\right)^{T}=\sum_{i=1}^{k} \mu_{i} e_{i}+\sum_{j=1}^{l} v_{j} d_{j} .
$$

The same logic in the bounded case holds, all slack variables of extreme points and extreme rays must be zero.

Finally, we have:

Theorem 4. Model R-Input $\mathrm{x}$ has optimal value zero if and only if Algorithm 1 outputs zero as the optimal value.

Proof. Recall that model R-Input $x$ has a linear objective function.

According to Proposition 1 and Proposition 2, $\mathcal{X}_{E}$ consists of some connected surface of a polyhedron. If we relax R-Input $x$ to an LP by taking the convex hull of $\mathcal{X}_{E}$, the optimal value and solution will stay the same, since optimal solutions of LP must lie on the surface of the corresponding feasible region. As a result, Algorithm 1 is a simplex algorithm for solving the relaxed LP of R-Input $x$, which completes the proof.

Remark 2. Algorithm 1 is also capable of generating the basis of all solutions with zero slacks. This can be achieved by changing the termination condition on line 9 to cache all neighbors when a corresponding objective is zero-valued.

\subsection{Numerical Examples}

In this subsection, we give two numerical examples to illustrate our revised model. As mentioned in the Introduction that Zhang and Cui [17] integrated inverse DEA models into 12 different cases, including the cases proposed by previous authors [11-16]. Therefore, we use the models in [17] to make a comparison. Note that existing inverse DEA models require a priori weight of each output. We default all the weights by one as [11], which is also a common practice in the literature.

\subsubsection{Example 1}

The dataset in Example 1, presented in Table 3, is the same as Table 1, where the inputs of $\mathrm{DMU}_{2}$ change and we need to find suitable outputs. Note that $\mathrm{DMU}_{2}$ has only zero slacks in Table 2, which means the desired outputs level should keep all slacks zero-valued. 
Table 3. Production Example 1.

\begin{tabular}{lccccc}
\hline & DMU $_{\mathbf{1}}$ & $\mathbf{D M U}_{\mathbf{2}}$ & $\mathbf{D M U}_{\mathbf{3}}$ & $\mathbf{D M U}_{\mathbf{4}}$ & $\mathbf{D M U}_{\mathbf{5}}$ \\
\hline Input 1 & 4 & 6 & 1 & 4 & 2 \\
Input 2 & 3 & 6 & 1 & 2 & 4 \\
Output 1 & 1 & 4 & 3 & 4 & 4 \\
Output 2 & 1 & 3.5 & 4 & 5 & 2 \\
\hline
\end{tabular}

\section{Case 1}

The inputs of $\mathrm{DMU}_{2}$ are altered to $\alpha_{1}=(7,5)$, and denote the desired outputs level as $\beta_{1}$. Label variable $\lambda_{i}, i=1, \ldots, 5$ and slack variables $s_{1}^{-}, s_{2}^{-}$sequentially as $1, \ldots, 7$, solving R-Input $\mathrm{X}$ by invoking Algorithm 1 . The programming is feasible, and $(3,4,6)$ is an initial basis that corresponds to efficient solution $x_{0}=(0,0,1 / 6,5 / 6,0,0,2 / 3)$, which is $\mathcal{C}=(3,4,6)$. The objective value of $e^{T} S^{-}$can still be improved by pivoting to an adjacent basis $(4,5,6)$ that corresponds to efficient solution $x_{1}=(0,0,0,3 / 4,1 / 4,0,0)$, where $e^{T} s^{-}=0$. Thus, we have reached to an optimal solution with $\beta_{1}=(4,17 / 4)$. The new DMU with production profile $\left(\alpha_{1}, \beta_{1}\right)$ has the radial efficiency index before change; moreover, it has no mix inefficiency.

On the contrary, if we employ the scalarization technique given in [17] to solve this case, it will result in $x_{0}$ with $\beta=(23 / 6,29 / 6), s^{-}=(0,2 / 3)$, which are not satisfying outputs to preserve mix inefficiency status.

\section{Case 2}

In this case, we let inputs of $\mathrm{DMU}_{2}$ change to $\alpha_{2}=(5,5)$ and denote the desired outputs level as $\beta_{2}$. Label variable $\lambda_{i}, i=1, \ldots, 5$ and slack variables $s_{1}^{-}, s_{2}^{-}$sequentially as $1, \ldots, 7$, solving R-Input by invoking Algorithm 1 . The programming is feasible, and by the same procedure in Case 1 the efficient solution is $x_{1}=(0,0,1 / 4,3 / 8,3 / 8,0,0)$, where $e^{T} s^{-}=0$. Thus, we have reached an optimal solution with $\beta_{2}=(15 / 4,29 / 8)$. The new DMU with production profile $\left(\alpha_{1}, \beta_{1}\right)$ has the radial efficiency index before change; moreover, it has no mix inefficiency.

However, the scalarization technique given in [17] will result in $x_{0}$ with $\beta=(7 / 2,9 / 2), s^{-}=(0,1)$, which are not satisfying outputs to preserve mix inefficiency status.

\section{Case 3}

For another case, if we let inputs of $\mathrm{DMU}_{2}$ change to $\alpha_{2}=(4,5 / 2)$, then the optimal solution of R-InputX is $(0,0,2 / 3,1 / 3,0,0,1 / 6)$ and the optimal objective value is no longer zero. This means that no output level can keep radial efficiency index invariant and no mix inefficiency at the same time.

The scalarization technique given in [17] results in $x_{0}$ with $\beta=(13 / 4,17 / 4), s^{-}=(1 / 4,0)$, neither of which are satisfying outputs that can preserve mix inefficiency status.

From the definition of $\mathcal{X}_{E}$, we can derive the expression for the predictable range of DMUs' inputs from $\left(\lambda, s^{-}\right)$in $\mathcal{X}_{E}$. We present the predictable range for all DMUs in Figure 1, it is worth mentioning that DMU1 is not predictable for its non-zero mix inefficiency. 


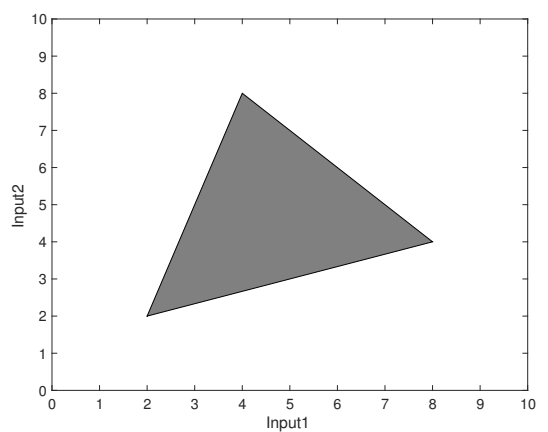

(a) DMU2

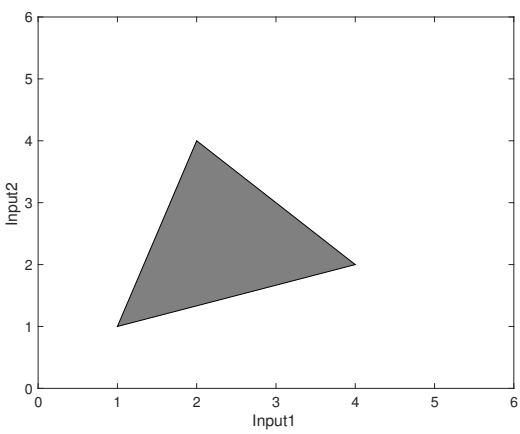

(b) DMU3-5

Figure 1. The predictable range of inputs on DMUs in Example 1.

\subsubsection{Example 2}

In this numerical example, we show that our algorithm may give several different satisfying predictions. We construct Example 2 by adding $\mathrm{DMU}_{6}$ and $\mathrm{DMU}_{7}$ to Example 1; the details are presented in Table 4. The DEA analysis results of this example are listed in Table 5; $\mathrm{DMU}_{3}-\mathrm{DMU}_{7}$ are strongly efficient, $\mathrm{DMU}_{1}$ is inefficient with non-zero slacks, $\mathrm{DMU}_{2}$ is inefficient with no slacks. Therefore, $\mathrm{DMU}_{1}$ is non-predictable and $\mathrm{DMU}_{3}-\mathrm{DMU}_{7}$ are predictable. The predictable range of inputs for $\mathrm{DMU}_{3}-\mathrm{DMU}_{7}$ are presented in Figure 2. Here, we still analyze the three cases of $\mathrm{DMU}_{2}$ in Example 1.

Table 4. Production Example 2.

\begin{tabular}{lccccccc}
\hline & DMU $_{\mathbf{1}}$ & $\mathbf{D M U}_{\mathbf{2}}$ & $\mathbf{D M U}_{\mathbf{3}}$ & $\mathbf{D M U}_{\mathbf{4}}$ & $\mathbf{D M U}_{\mathbf{5}}$ & $\mathbf{D M U}_{\mathbf{6}}$ & $\mathbf{D M U}_{\mathbf{7}}$ \\
\hline Input 1 & 4 & 6 & 1 & 4 & 2 & 3 & 1.5 \\
Input 2 & 3 & 6 & 1 & 2 & 4 & 1.5 & 3 \\
Output 1 & 1 & 4 & 3 & 4 & 4 & 1 & 4 \\
Output 2 & 1 & 3.5 & 4 & 5 & 2 & 5 & 1 \\
\hline
\end{tabular}

Table 5. Results of two phase procedure.

\begin{tabular}{lccccccc}
\hline & $\mathbf{D M U}_{\mathbf{1}}$ & $\mathbf{D M U}_{\mathbf{2}}$ & $\mathbf{D M U}_{\mathbf{3}}$ & $\mathbf{D M U}_{\mathbf{4}}$ & $\mathbf{D M U}_{\mathbf{5}}$ & $\mathbf{D M U}_{\mathbf{6}}$ & $\mathbf{D M U}_{\mathbf{7}}$ \\
\hline Efficiency & $1 / 3$ & $1 / 2$ & 1 & 1 & 1 & 1 & 1 \\
$s_{1}^{-}$ & $1 / 3$ & 0 & 0 & 0 & 0 & 0 & 0 \\
$s_{2}^{-}$ & 0 & 0 & 0 & 0 & 0 & 0 & 0 \\
$s_{1}^{+}$ & 2 & 0 & 0 & 0 & 0 & 0 & 0 \\
$s_{2}^{+}$ & 3 & 0 & 0 & 0 & 0 & 0 & 0 \\
\hline
\end{tabular}

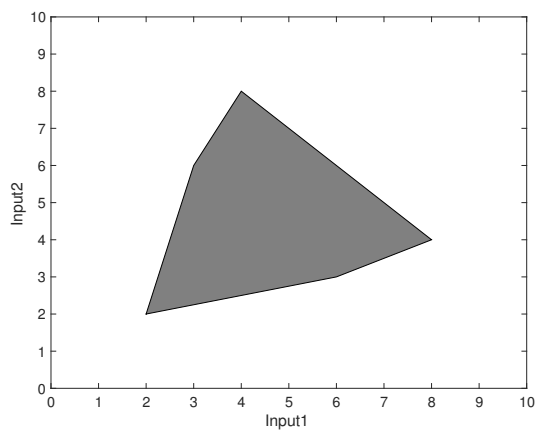

(a) DMU2

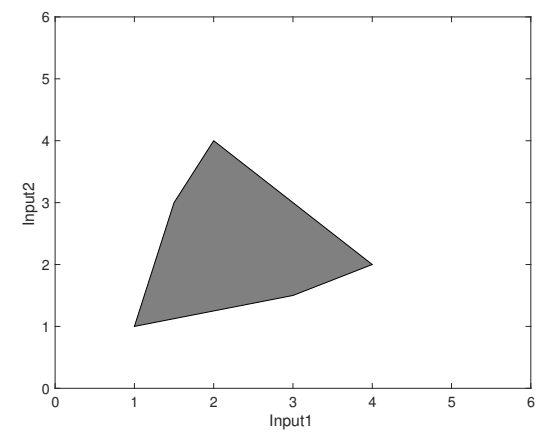

(b) DMU3-7

Figure 2. The predictable range of inputs on DMUs in Example 2. 


\section{Case 1}

The inputs of $\mathrm{DMU}_{2}$ are altered to $\alpha_{1}=(7,5)$, and denote the desired outputs level as $\beta_{1}^{\prime}$. Label variable $\lambda_{i}, i=1, \ldots, 7$ and slack variables $s_{1}^{-}, s_{2}^{-}$sequentially as $1, \ldots, 9$, solving R-Input $\mathrm{X}$ by invoking Algorithm 1. The programming is feasible, and the efficient solution is unique, which is $x_{1}=(0,0,0,3 / 4,1 / 4,0,0,0,0)$ with $e^{T} s^{-}=0$. Thus, we have reached an optimal solution with $\beta_{1}^{\prime}=(4,17 / 4)$. The new DMU with production profile $\left(\alpha_{1}, \beta_{1}^{\prime}\right)$ has the radial efficiency index before change; moreover, it has no mix inefficiency.

The scalarization technique given in [17] results in $x_{0}$ with $\beta=(23 / 6,29 / 6), s^{-}=(0,2 / 3)$, which are not satisfying outputs to preserve mix inefficiency status.

\section{Case 2}

The inputs of $\mathrm{DMU}_{2}$ are altered to $\alpha_{2}=(5,5)$, and denote the desired outputs level as $\beta_{2}^{\prime}$. Label variable $\lambda_{i}, i=1, \ldots, 7$ and slack variables $s_{1}^{-}, s_{2}^{-}$sequentially as $1, \ldots, 9$, solving R-Input $x$ by invoking Algorithm 1. The programming is feasible, and by the same procedure in the Case 1 of Example 1, the extreme points of all the efficient solution are $(0,0,1 / 4,3 / 8,3 / 8,0,0,0,0)$, $(0,0,1 / 22,9 / 22,0,0,6 / 11,0,0),(0,0,1 / 22,0,9 / 22,6 / 11,0,0,0)$, where $e^{T} s^{-}=0$. Thus, we have reached optimal solutions, which are the convex combination of $(15 / 4,29 / 8),(87 / 22,30 / 11)$, $(51 / 22,41 / 11)$. The new DMU has the same radial efficiency index before change; moreover, it has no mix inefficiency.

The scalarization technique given in [17] results in $x_{0}$ with $\beta=(7 / 2,9 / 2), s^{-}=(0,1)$, which are not satisfying outputs to preserve mix inefficiency status.

\section{Case 3}

If we let inputs of $\mathrm{DMU}_{2}$ change to $\alpha_{3}=(4,5 / 2)$, then the optimal solution of R-Input $\mathrm{X}$ is $(0,0,1 / 2,0,0,1 / 2,0,0,0)$ with $e^{T} s^{-}=0$. Therefore, we reach an optimal solution with $\beta_{3}^{\prime}=(2,9 / 2)$. It is worth mentioning that, with more information $\left(\mathrm{DMU}_{6}, \mathrm{DMU}_{7}\right)$ given, $\alpha_{3}$ become predictable in this example.

The scalarization technique given in [17] results in $x_{0}$ with $\beta=(13 / 4,17 / 4)$, $\mathrm{s}^{-}=(1 / 4,0)$, which are not satisfying outputs to preserve mix inefficiency status.

\subsection{Discussion}

The numerical examples are summarized in Table 6, where 'Revised' stands for the inverse DEA model R-Inputx. The mix inefficiency of DMU2 in the two phase procedure was zero before the changes in both examples, therefore the efficiency level has been preserved. In contrast, inverse DEA model in [17] will result in a new DMU with non-zero mix inefficiency. According to Remark 1 , the efficiency level of DMU2 has been changed.

Table 6. Comparison of our algorithms and the previous inverse radial model [17]. Revised: our inverse DEA model R-Input $x$. Conv: convex combination.

\begin{tabular}{llcccccc}
\hline \multirow{2}{*}{ DMU2 } & \multicolumn{2}{c}{$\mathbf{( 7 , 5 )}$} & \multicolumn{2}{c}{$(5,5)$} & \multicolumn{2}{c}{$\mathbf{( 4 , 5 / 2 )}$} \\
\cline { 2 - 8 } & & Revised & {$[17]$} & Revised & {$[17]$} & B & {$[17]$} \\
\hline \multirow{2}{*}{ Example 1 } & Output & $(4,17 / 4)$ & $(23 / 6,29 / 6)$ & $(15 / 4,29 / 8)$ & $(7 / 2,9 / 2)$ & - & $(13 / 4,17 / 4)$ \\
\cline { 2 - 8 } & Mix inefficiency & $(0,0)$ & $(0,2 / 3)$ & $(0,0)$ & $(0,1)$ & - & $(1 / 4,0)$ \\
\hline \multirow{4}{*}{ Example 2 } & \multirow{2}{*}{ Output } & $(4,17 / 4)$ & $(23 / 6,29 / 6)$ & $\begin{array}{c}\text { Conv }((15 / 4,29 / 8), \\
(87 / 22,30 / 11), \\
(51 / 22,41 / 11))\end{array}$ & $(7 / 2,9 / 2)$ & $(2,9 / 2)$ & $(13 / 4,17 / 4)$ \\
\cline { 2 - 8 } & Mix inefficiency & $(0,0)$ & $(0,2 / 3)$ & $(0,0)$ & $(0,1)$ & $(0,0)$ & $(1 / 4,0)$ \\
\hline
\end{tabular}

Aside from its ability to preserve the overall efficiency, the inverse DEA model R-Input $x$ can provide the user with more objective information about the production possibilities. One of the 
advantages of the DEA model is that it avoids prior assumptions about relative weights of each input and output while accessing DMUs' relative efficiency. The weights prescribed by the DEA model are derived directly from the data and each DMU is evaluated in its best possible light. This feature makes DEA an objective efficiency evaluation method. Although there are techniques that impose control over the weights, a certain level of flexibility of the weight is still preserved. As we mentioned in Remark 2, our model can give the basis of all satisfying output combinations without the need to prescribe the weights. For example, in Case 2 in Example 2, after the inputs for DMU2 are changed to $(5,5)$, our model computes three basis solutions: $(15 / 4,29 / 8),(87 / 22,30 / 11),(51 / 22,41 / 11)$, and any linear combination of these solutions leads to a DMU with the efficiency level before the inputs change. However, if we use the technique in [17] to solve the inverse DEA model, the relative weights of the DMU after input change are also specified, which means that the user of the inverse DEA model can manipulate the weights according to his or her preference. In such cases, the results given by the inverse DEA model are no longer impartial.

However, there are some limitations of this revised model R-Inputx. First of all, it is specially designed for DMUs without mix inefficiency, whereas non-zero slacks are quite common in practice. However, as we argued earlier, this is the limitation of radial DEA models. Nevertheless, as model R-Inputx tries to minimize slacks, it can be used to find an approximation result when the mix inefficiencies are required to be relatively small before and after the change. This result only implies that no output level can preserve the overall efficiency of $\mathrm{DMU}_{\mathrm{o}}$ under the current $\mathrm{DEA}$ model. Another limitation of the model R-Inputx is that, even for DMUs without mix inefficiency, the predictable range for the DMUs is limited. However, as illustrated in the numerical experiments, the more information we are given, the larger range we can predict, which is consistent with our perception.

\section{Conclusions}

This paper points out possible failures of existing radial inverse DEA models and provides a remedy for some situations. Although classic radial DEA models evaluate the total efficiency level of DMUs through both the radial efficiency index and the mix inefficiency, existing radial inverse DEA models consider only radial efficiency index. As a consequence, current radial inverse DEA models may result in a new DMU with different efficiency levels, breaching the assumption of inverse DEA.

We assess the influence of this kind of inconsistency by giving a counterexample where a radial inverse DEA model predicts outputs to increase while inputs decrease. This kind of failure is inevitable when slacks exist and will render inverse DEA models unreliable. Because of these drawbacks, we suggest that the results given by inverse DEA models should be interpreted and used with care.

To rectify this problem, we revise radial inverse DEA models for a particular situation where DMU of interest has no mix inefficiency, that is, radial efficiency index alone can represent the total efficiency level. The revised model is developed by excluding all mix inefficiency during the change while preserving radial efficiency index. Our model can redeem the unaltered efficiency level and can be solved efficiently using a simplex-like algorithm. Given that standard radial DEA models do not have a unified measure of efficiency level, it is not clear how to develop inverse DEA models that apply to general cases.

In our numerical experiments, we find the traditional inverse DEA may manipulate the weights according to personal preference, which introduces a priori assumptions about relative weights of each output and violates the property of DEA. In contrast, our model avoids prior assumptions about relative weights of each input and output while accessing DMUs' relative efficiency. Therefore, our algorithm computes all Pareto solutions for our test examples, where the mix inefficiency is avoided.

Finally, it would be an important direction to develop inverse models for non-radial models as well as an efficient algorithm to solve it, especially models that have a unified efficiency measure. 
Author Contributions: Conceptualization, methodology, software, validation, formal analysis, investigation, resources, and writing-original draft preparation, X.H. and J.L.; writing-review and editing, X.L.; supervision, project administration, and funding acquisition, J.C. All authors have read and agreed to the published version of the manuscript.

Funding: This research received no external funding.

Acknowledgments: This work is supported by Key Laboratory of Management, Decision and Information Systems, CAS.

Conflicts of Interest: The authors declare no conflict of interest.

\section{Abbreviations}

The following abbreviations are used in this manuscript:

$\begin{array}{ll}\text { BES } & \text { Basic efficient solution } \\ \text { BFS } & \text { Basic feasible solution } \\ \text { CCR } & \text { Charnes, Cooper, and Rhodes } \\ \text { CRS } & \text { Constant Return to Scale } \\ \text { DEA } & \text { Data envelopment analysis } \\ \text { DMU } & \text { Decision making units } \\ \text { EB } & \text { Efficient basis } \\ \text { FB } & \text { Feasible bases } \\ \text { LP } & \text { Linear programming } \\ \text { MOLP } & \text { Multi-objective linear programming } \\ \text { NDRS } & \text { Non-decreasing return to scale } \\ \text { NIRS } & \text { Non-increasing return to scale } \\ \text { VRS } & \text { Various return to Scale }\end{array}$

\section{References}

1. Liu, J.S.; Lu, L.Y.; Lu, W.M.; Lin, B.J. A survey of DEA applications. Omega 2013, 41, 893-902. [CrossRef]

2. Puig-Junoy, J. Partitioning input cost efficiency into its allocative and technical components: An empirical DEA application to hospitals. Socio-Econ. Plan. Sci. 2000, 34, 199-218. [CrossRef]

3. Yang, W.; Cai, L.; Edalatpanah, S.A.; Smarandache, F. Triangular Single Valued Neutrosophic Data Envelopment Analysis: Application to Hospital Performance Measurement. Symmetry 2020, 12, 588. [CrossRef]

4. Popović, M.; Savić, G.; Kuzmanović, M.; Martić, M. Using Data Envelopment Analysis and Multi-Criteria Decision-Making Methods to Evaluate Teacher Performance in Higher Education. Symmetry 2020, 12, 563. [CrossRef]

5. Krmac, E.; Djordjević, B. A New DEA Model for Evaluation of Supply Chains: A Case of Selection and Evaluation of Environmental Efficiency of Suppliers. Symmetry 2019, 11, 565. [CrossRef]

6. Xu T., You J.; Shao, Y. Efficiency of China's Listed Securities Companies: Estimation through a DEA-Based Method. Mathematics 2020, 8, 589. [CrossRef]

7. Zhang, J.; Wang, S.; Yang, P.; Fan, F.; Wang, X. Analysis of Scale Factors on China's Sustainable Development Efficiency Based on Three-Stage DEA and a Double Threshold Test. Sustainability 2020, 12, 2225. [CrossRef]

8. Kamarudin, F.; Sufian, F.; Nassir, A.M.; Anwar, N.A.M.; Hussain, H.I. Bank efficiency in Malaysia a DEA approach. J. Cent. Bank. Theory Pract. 2019, 8, 133-162. [CrossRef]

9. Yu, M.M.; Lin, C.I.; Chen, K.C.; Chen, L.H. Measuring Taiwanese bank performance: A two-system dynamic network data envelopment analysis approach. Omega 2019, 102145. [CrossRef]

10. Charnes, A.; Cooper, W.W.; Rhodes, E. Measuring the efficiency of decision-making units. Eur. J. Oper. Res. 1978, 2, 429-444. [CrossRef]

11. Wei, Q.; Zhang, J.; Zhang, X. An inverse DEA model for inputs/outputs estimate. Eur. J. Oper. Res. 2000, 121, 151-163. [CrossRef]

12. Zhang, X.S.; Cui, J.C. A project evaluation system in the state economic information system of China an operations research practice in public sectors. Int. Trans. Oper. Res. 1999, 6, 441-452. [CrossRef]

13. Jahanshahloo, G.R.; Hosseinzadeh Lotfi, F.; Shoja, N.; Tohidi, G.; Razavyan, S. Input estimation and identification of extra inputs in inverse DEA models. Appl. Math. Comput. 2004, 156, 427-437. [CrossRef]

14. Hadi-Vencheh, A.; Foroughi, A.A. A generalized DEA model for inputs/outputs estimation. Math. Comput. Model. 2006, 43, 447-457. [CrossRef] 
15. Hadi-Vencheh, A.; Foroughi, A.A.; Soleimani-damaneh, M. A DEA model for resource allocation. Econ. Model. 2008, 25, 983-993. [CrossRef]

16. Lertworasirikul, S.; Charnsethikul, P.; Fang, S.C. Inverse data envelopment analysis model to preserve relative efficiency values: The case of variable returns to scale. Comput. Ind. Eng. 2011, 61, 1017-1023. [CrossRef]

17. Zhang, M.; Cui, J.C. The extension and integration of the inverse DEA method. J. Oper. Res. Soc. $2016,67$. [CrossRef]

18. Yan, H.; Wei, Q.; Hao, G. DEA models for resource reallocation and production input/output estimation. Eur. J. Oper. Res. 2002, 136, 19-31. [CrossRef]

19. Jahanshahloo, G.R.; Hadi Vencheh, A.; Foroughi, A.A.; Kazemi Matin, R. Inputs/outputs estimation in DEA when some factors are undesirable. Appl. Math. Comput. 2004, 156, 19-32. [CrossRef]

20. Eyni, M.; Tohidi, G.; Mehrabeian, S. Applying inverse DEA and cone constraint to sensitivity analysis of DMUs with undesirable inputs and outputs. J. Oper. Res. Soc. 2017, 68, 34-40. [CrossRef]

21. Ghiyasi, M. Inverse DEA based on cost and revenue efficiency. Comput. Ind. Eng. 2017, 114, $258-263$. [CrossRef]

22. Jahanshahloo, G.R.; Hosseinzadeh Lotfi, F.; Shoja, N.; Tohidi, G.; Razavyan, S. The outputs estimation of a DMU according to improvement of its efficiency. Appl. Math. Comput. 2004, 147, 409-413. [CrossRef]

23. Lim, D.J. Inverse DEA with frontier changes for new product target setting. Eur. J. Oper. Res. 2016, 254, 510-516. [CrossRef]

24. Lin, H.T. An efficiency-driven approach for setting revenue target. Decis. Support Syst. 2010, 49, 311-317. [CrossRef]

25. Frija, A.; Wossink, A.; Buysse, J.; Speelman, S.; Van Huylenbroeck, G. Irrigation pricing policies and its impact on agricultural inputs demand in Tunisia: A DEA-based methodology. J. Environ. Manag. 2011, 92, 2109-2118. [CrossRef]

26. Gattoufi, S.; Amin, M.; Emrouznejad, A. A new inverse DEA method for merging banks. IMA J. Manag. Math. 2014, 25. [CrossRef]

27. Amin, G.R.; Emrouznejad, A.; Gattoufi, S. Modelling generalized firms' restructuring using inverse DEA. J. Product. Anal. 2017, 48, 51-61. [CrossRef]

28. Amin, G.R.; Emrouznejad, A.; Gattoufi, S. Minor and major consolidations in inverse DEA: Definition and determination. Comput. Ind. Eng. 2017, 103, 193-200. [CrossRef]

29. Feng, C.; Chu, F.; Ding, J.; Bi, G.; Liang, L. Carbon Emissions Abatement (CEA) allocation and compensation schemes based on DEA. Omega 2015, 53, 78-89. [CrossRef]

30. Emrouznejad, A.; Yang, G.L.; Amin, G.R. A novel inverse DEA model with application to allocate the $\mathrm{CO} 2$ emissions quota to different regions in Chinese manufacturing industries. J. Oper. Res. Soc. 2019, 70, 1079-1090. [CrossRef]

31. Hassanzadeh, A.; Yousefi, S.; Saen, R.F.; Hosseininia, S.S.S. How to assess sustainability of countries via inverse data envelopment analysis? Clean Technol. Environ. Policy 2018, 20, 29-40. [CrossRef]

32. Jahanshahloo, G.R.; Hosseinzadeh Lotfi, F.; Rostamy-Malkhalifeh, M.; Ghobadi, S. Using Enhanced Russell Model to Solve Inverse Data Envelopment Analysis Problems. Sci. World J. 2014, 2014, 671896. [CrossRef] [PubMed]

33. Hu, X.; Li, J.; Li, X.; Cui, J. Carbon Emissions Abatement (CEA) Allocation Based on Inverse Slack-Based Model (SBM). J. Oper. Res. Soc. China 2020. [CrossRef]

34. Cooper, W.W.; Seiford, L.M.; Tone, K. Data Envelopment Analysis: A Comprehensive Text with Models, Applications, References and DEA-Solver Software, 2nd ed.; Springer: New York, NY, USA, 2007.

35. Evans, J.P.; Steuer, R.E. A revised simplex method for linear multiple objective programs. Math. Program. 1973, 5, 54-72. [CrossRef]

36. Yu, P.L.; Zeleny, M. The set of all nondominated solutions in linear cases and a multicriteria simplex method. J. Math. Anal. Appl. 1975, 49, 430-468. [CrossRef]

37. Steuer, R.E. Multiple Criteria Optimization: Theory, Computation, and Application; Google-Books-ID: tSA_PgAACAAJ; Krieger: Baltimore, MD, USA, 1989. 
38. Benson, H.P. Finding an Initial Efficient Extreme Point for a Linear Multiple Objective Program. J. Oper. Res. Soc. 1981, 32, 495-498. [CrossRef]

39. Bland, R.G. New Finite Pivoting Rules for the Simplex Method. Math. Oper. Res. 1977, 2, 103-107 [CrossRef]

(C) 2020 by the authors. Licensee MDPI, Basel, Switzerland. This article is an open access article distributed under the terms and conditions of the Creative Commons Attribution (CC BY) license (http://creativecommons.org/licenses/by/4.0/). 